\title{
Solutions of renormalization group flow equations with full momentum dependence
}

\author{
F. Benitez,${ }^{1}$ J.-P. Blaizot ${ }^{2}$ H. Chaté,${ }^{3}$ B. Delamotte, ${ }^{4}$ R. Méndez-Galain,${ }^{1}$ and N. Wschebor ${ }^{1}$ \\ ${ }^{1}$ Instituto de Fisica, Faculdad de Ingeniería, Universidade de la República, 11000 Montevideo, Uruguay \\ ${ }^{2}$ IPhT, CEA-Saclay, 91191 Gif-sur-Yvette, France \\ ${ }^{3}$ Service de Physique de l'Etat Condensé, CEA-Saclay, 91191 Gif-sur-Yvette, France \\ ${ }^{4}$ LPTMC, CNRS-UMR 7600, Université Pierre et Marie Curie, 75252 Paris, France
}

(Dated: October 31, 2018)

\begin{abstract}
We demonstrate the power of a recently-proposed approximation scheme for the non-perturbative renormalization group that gives access to correlation functions over their full momentum range. We solve numerically the leading-order flow equations obtained within this scheme, and compute the two-point functions of the $O(N)$ theories at criticality, in two and three dimensions. Excellent results are obtained for both universal and non-universal quantities at modest numerical cost.
\end{abstract}

PACS numbers: 05.10.Cc,64.60.ae,11.10.Hi

The renormalization group, in its non-perturbative version [1, 2] (also referred to as the exact renormalization group), provides a general formalism giving access, for arbitrary coupling strength, to a whole set of physically important quantities, universal as well as non-universal [3, 4], thermodynamic functions and momentum-dependent correlation functions, etc. However, most studies within this framework involve approximations that restrict their scope to the calculation of thermodynamical quantities, or correlation functions with vanishing external momenta. In order to access the full momentum dependence, Blaizot, Méndez-Galain, and Wschebor (BMW) have recently introduced an approximation scheme which overcomes this limitation [5]. In principle, this scheme allows to compute, in all dimensions, at and away from criticality, both universal and non-universal quantities, as well as momentumdependent properties from $p=0$ up to the ultra-violet cut-off $\Lambda$ (inverse lattice spacing).

In this Letter, we present the first complete implementation of the leading order approximation of the BMW scheme, and demonstrate its power by using $O(N)$ models as a testbed. We compute the entire momentum dependence of the two-point functions in two and three dimensions and obtain excellent results for both universal and non-universal quantities.

We start by a brief outline of the formalism. In order to simplify the presentation, we shall write only the equations corresponding to the case of a scalar field theory with quartic coupling, i.e., restrict the presentation to the case $N=1$ (corresponding to the Ising model). The strategy of the renormalization group is to build a family of theories indexed by a momentum scale parameter $k$, such that fluctuations are smoothly taken into account as $k$ is lowered from the microscopic scale $\Lambda$ down to 0 . In practice, this is achieved by adding to the original Euclidean action $S$ a mass-like term of the form $\Delta S_{k}[\varphi]=\frac{1}{2} \int_{q} R_{k}\left(q^{2}\right) \varphi(q) \varphi(-q)$. The cut-off function $R_{k}\left(q^{2}\right)$ is chosen so that $R_{k}\left(q^{2}\right) \sim k^{2}$ for $q \lesssim k$, which effectively suppresses the modes $\varphi(q \lesssim k)$, and so that it vanishes for $q \gtrsim k$, leaving the modes $\varphi(q \gtrsim k)$ unaffected. One then defines a scale-dependent partition function

$$
\mathcal{Z}_{k}[J]=\int \mathcal{D} \varphi e^{-S[\varphi]-\Delta S_{k}[\varphi]+\int J \varphi},
$$

and a scale-dependent effective action $\Gamma_{k}[\phi]$ through a (slightly modified) Legendre transform [2],

$$
\Gamma_{k}[\phi]+\log \mathcal{Z}_{k}[J]=\int J \phi-\frac{1}{2} \int_{q} R_{k}\left(q^{2}\right) \phi_{q} \phi_{-q},
$$

with $\phi=\delta \ln \mathcal{Z}_{k} / \delta J$. The variation of the effective action $\Gamma_{k}[\phi]$ as $k$ varies is governed by Wetterich's equation [1]:

$$
\partial_{k} \Gamma_{k}=\frac{1}{2} \int_{q} \partial_{k} R_{k}\left(q^{2}\right) G_{k}[q, \phi],
$$

where $G_{k}[q, \phi]=\left(\Gamma_{k}^{(2)}[q, \phi]+R_{k}\left(q^{2}\right)\right)^{-1}$, and $\Gamma_{k}^{(2)}[q, \phi]$ is the second functional derivative of $\Gamma_{k}[\phi]$ w.r.t. $\phi$. The initial conditions of the flow equation (3) correspond to the microscopic scale $k=\Lambda$ where all fluctuations are frozen by the $\Delta S_{k}$ term, so that $\Gamma_{k=\Lambda}[\phi]=S[\phi]$. The effective action of the original theory is obtained as the solution of (3) for $k \rightarrow 0$ where $R_{k}\left(q^{2}\right)$ vanishes. Differentiating Eq. (3) $m$ times with respect to $\phi$ yields the flow equation for the vertex function $\Gamma_{k}^{(m)}\left[q_{1}, \ldots, q_{m} ; \phi\right]$. Thus for instance, the flow equation for $\Gamma^{(2)}$ reads:

$$
\begin{gathered}
\partial_{k} \Gamma_{k}^{(2)}(p)=\int_{q} \partial_{k} R_{k}\left(q^{2}\right) G_{k}^{2}(q)\left[\Gamma_{k}^{(3)}(p,-p-q, q) \times\right. \\
\left.G_{k}(p+q) \Gamma_{k}^{(3)}(-p, p+q,-q)-\frac{1}{2} \Gamma_{k}^{(4)}(p,-p, q,-q)\right] .
\end{gathered}
$$

(Here we assumed the field $\phi$ to be uniform, and omitted the $\phi$ dependence to alleviate the notation.) Note that the flow equation for $\Gamma_{k}^{(m)}\left[q_{1}, \ldots, q_{m} ; \phi\right]$ involves $\Gamma_{k}^{(m+1)}$ and $\Gamma_{k}^{(m+2)}$, leading to an infinite hierarchy.

The flow equation (3), and the equivalent flow equations for the vertex functions, are exact, but their solution requires, in general, approximations. It is precisely one of the virtues of this formulation of field theory to 
suggest approximation schemes that are not easily derived in other, more conventional approaches. In particular, one can develop approximation schemes for the effective action itself, that is, which apply to the entire set of correlation functions. The BMW approximation [5] is such a scheme. It relies on two observations. First, the presence of the cut-off function $R_{k}\left(q^{2}\right)$ insures the smoothness of the $\Gamma_{k}^{(m)}$, s and limits the internal momentum $q$ in equations such as Eq. (4) to $q \lesssim k$. In line with this observation, one neglects the $q$-dependence of the vertex functions in the r.h.s. of the flow equations (e.g. in $\Gamma^{(3)}$ and $\Gamma^{(4)}$ in Eq. (4)), while keeping the full dependence on the external momenta $p_{i}$. The second observation is that, for uniform fields, $\Gamma_{k}^{(m+1)}\left(p_{1}, \ldots, p_{m}, 0, \phi\right)=$ $\partial_{\phi} \Gamma_{k}^{(m)}\left(p_{1}, \ldots, p_{m}, \phi\right)$, which enables one to close the hierarchy of equations.

At the leading order of the BMW scheme one keeps the non trivial momentum dependence of the two-point function and implements the approximations above on Eq. (4), which becomes:

$$
k \partial_{k} \Gamma_{k}^{(2)}(p, \phi)=J_{3}(p, \phi)\left(\partial_{\phi} \Gamma_{k}^{(2)}\right)^{2}-\frac{1}{2} J_{2}(0, \phi) \partial_{\phi}^{2} \Gamma_{k}^{(2)}
$$

with

$$
J_{n}(p, \phi) \equiv \int_{q} k \partial_{k} R_{k}\left(q^{2}\right) G_{k}^{n-1}(q, \phi) G_{k}(p+q, \phi) .
$$

The approximation can be systematically improved: The order $m$ consists in keeping the full momentum dependence of $\Gamma_{k}^{(2)}, \ldots, \Gamma_{k}^{(m)}$ and truncating that of $\Gamma_{k}^{(m+1)}$ and $\Gamma_{k}^{(m+2)}$ along the same lines as those leading to Eq. (5) corresponding to $m=2$.

The zeroth order approximation is the so-called local potential approximation (LPA) where vertex functions are obtained as derivatives of the effective potential $V_{k}$ (equal, to within a volume factor, to $\Gamma_{k}$ evaluated for a uniform $\phi), \Gamma_{k}^{(m)}\left(p_{1}, \cdots, p_{m}, \phi\right) \stackrel{\text { LPA }}{=} V_{k}^{(m)}(\phi)$, except for $\Gamma_{k}^{(2)}(p, \phi) \stackrel{\text { LPA }}{=} p^{2}+V_{k}^{(2)}(\phi)$. The LPA has been widely used with reasonable success [2, 4, 6, 7]. It can be improved through a systematic expansion in gradients of the fields, usually referred to as the derivative expansion (DE) [2, 8]. However, in contrast to the BMW scheme, the DE, at any finite order, does not give access to correlation functions with non-vanishing external momenta (or with external momenta larger than the smallest mass).

We now turn to the main purpose of the present Letter, which is to show that the nonlinear integro-partialdifferential equation (5) can be studied as is, without further approximation [9]. Note that the earlier studies of Eq. (5) presented in [11] involve additional approximations which are linked to a specific cut-off function, and which become too crude below three dimensions.

In order to treat efficiently the low (including zero) momentum sector, we work with dimensionless and renormalized quantities. Thus, we measure all momenta in units of $k: \tilde{p}=p / k$. We also rescale $\rho \equiv \frac{1}{2} \phi^{2}$ according to $\tilde{\rho}=k^{2-d} Z_{k} K_{d}^{-1} \rho\left(K_{d}=(2 \pi)^{-d} S_{d} / d, S_{d}\right.$ being the volume of the unit sphere), and set $\tilde{\Gamma}_{k}^{(2)}(\tilde{p}, \tilde{\rho})=$ $k^{-2} Z_{k}^{-1} \Gamma_{k}^{(2)}(p, \rho)$. The running anomalous dimension $\eta_{k}$ is defined by $k \partial_{k} Z_{k}=-\eta_{k} Z_{k}$, so that at a fixed point $Z_{k} \sim k^{-\eta}, \eta$ being the anomalous dimension of the field at the fixed point. The absolute normalization of $Z_{k}$ is fixed by choosing a point $\left(\tilde{p}_{0}, \tilde{\rho}_{0}\right)$ where $\left.\partial_{\tilde{p}^{2}} \tilde{\Gamma}_{k}^{(2)}\right|_{\tilde{p}_{0}, \tilde{\rho}_{0}}=1$. Then, the flow equation of $\tilde{\Gamma}_{k}^{(2)}(\tilde{p}, \tilde{\rho})$ follows trivially from Eq.(5). For numerical reasons, we actually solve two equations: one for $\tilde{Y}_{k}(\tilde{p}, \tilde{\rho}) \equiv \tilde{p}^{-2}\left[\tilde{\Gamma}_{k}^{(2)}(\tilde{p}, \tilde{\rho})-\tilde{\Gamma}_{k}^{(2)}(0, \tilde{\rho})\right]-$ 1 and one for the derivative of the dimensionless effective potential $\tilde{W}_{k}(\tilde{\rho})=Z_{k}^{-1} k^{-2} \partial_{\rho} V_{k}(\rho)$. Note that $\tilde{\Gamma}_{k}^{(2)}(0, \tilde{\rho})=\tilde{W}_{k}(\tilde{\rho})+2 \tilde{\rho} \tilde{W}_{k}^{\prime}(\tilde{\rho})$. (Here and below, primes denote derivative w.r.t. $\tilde{\rho}$.) These two equations read (dropping the $k$ index to simplify the notation):

$$
\begin{aligned}
\partial_{t} \tilde{Y}= & \eta_{k}(1+\tilde{Y})+\tilde{p} \partial_{\tilde{p}} \tilde{Y}-\left(2-d-\eta_{k}\right) \tilde{\rho} \tilde{Y}^{\prime} \\
& +2 \tilde{\rho} \tilde{p}^{-2}\left[\left(\tilde{p}^{2} \tilde{Y}^{\prime}+\tilde{\lambda}_{k}\right)^{2} \tilde{J}_{3}(\tilde{p}, \tilde{\rho})-\tilde{\lambda}_{k}^{2} \tilde{J}_{3}(0, \tilde{\rho})\right] \\
& -\tilde{J}_{2}(0, \tilde{\rho})\left(\tilde{Y}^{\prime} / 2+\tilde{\rho} \tilde{Y}^{\prime \prime}\right) \\
\partial_{t} \tilde{W}= & \left(\eta_{k}-2\right) \tilde{W}+\left(d-2+\eta_{k}\right) \tilde{\rho} \tilde{W}^{\prime}+\frac{1}{2} \tilde{J}_{1}^{\prime}(0, \tilde{\rho})
\end{aligned}
$$

Here $\partial_{t}=k \partial_{k}, \eta_{k}$ is obtained by setting $\tilde{Y}_{k}\left[\tilde{p}_{0}, \tilde{\rho}_{0}\right]=$ 0 in Eq.(7), $\tilde{J}_{n}(\tilde{p}, \tilde{\rho})=K_{d}^{-1} Z_{k}^{n-1} k^{2 n-d-2} J_{n}(p, \rho)$ and $\tilde{\lambda}_{k}(\tilde{\rho})=3 \tilde{W}_{k}^{\prime}(\tilde{\rho})+2 \tilde{\rho} \tilde{W}_{k}^{\prime \prime}(\tilde{\rho})$.

In practice, we use a fixed, regular, $(\tilde{p}, \tilde{\rho})$ grid and restrict the range of the cut-off function by setting $R_{k}(\tilde{q} \geq 4)=0$. When computing the double integrals $\tilde{J}_{3}(\tilde{p}, \tilde{\rho})$, we need to evaluate $\tilde{Y}$ for momenta $\tilde{p}+\tilde{q}$ beyond $\tilde{p}_{\max }$, the maximal value on the grid. In such cases, we set $\tilde{Y}(\tilde{p})=\tilde{Y}\left(\tilde{p}_{\max }\right)$, an approximation checked to be excellent for $\tilde{p}_{\max } \geq 5$. To access the full momentum dependence, we also calculate $\Gamma_{k}^{(2)}(p, \tilde{\rho})$ at a set of fixed, freely chosen, external $p$ values. For a given such $p, p / k$ is within the grid at the beginning of the flow. This is no longer so when $k<p / \tilde{p}_{\text {max }}$; then, we switch to the dimensionful version of (7), and also set $J_{3}(p, \tilde{\rho})=G(p, \tilde{\rho}) J_{2}(0, \tilde{\rho})$, an excellent approximation when $p>k \tilde{p}_{\max }$.

We found that the simplest time-stepping (explicit Euler), a finite-difference evaluation of derivatives on a regular $(\tilde{p}, \tilde{\rho})$ grid, and the use of Simpson's rule to calculate integrals, are sufficient to produce stable and fastconverging results. For all the quantities calculated, the convergence to three significant digits is reached with a $(\tilde{p}, \tilde{\rho})$ grid of $50 \times 60$ points; with such a grid, a typical run takes a few minutes on a current personal computer.

Physical quantities exhibit a small dependence on the shape of $R_{k}\left(q^{2}\right)$ and on the point $\left(\tilde{p}_{0}, \tilde{\rho}_{0}\right)$ where $\eta_{k}$ is computed. Since in the absence of any approximation, they would be strictly independent of the cut-off function and of the choice of the renormalization point, a study of this spurious dependence provides an indica- 
(a)

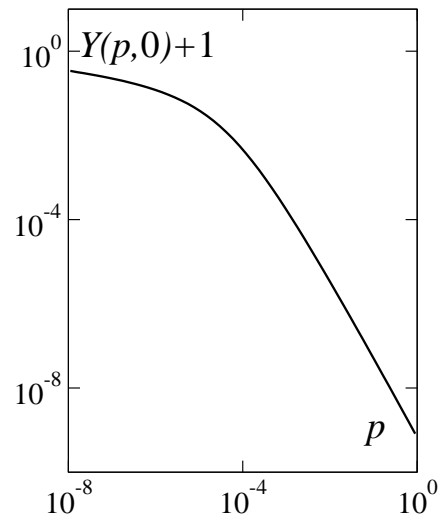

(b)

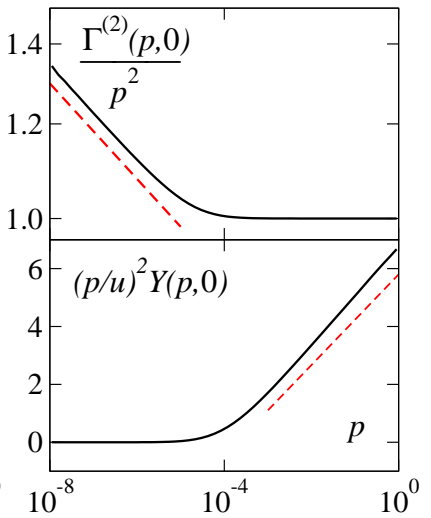

FIG. 1: (Color online) Typical results at criticality and $k=$ 0 for $N=2$ in $d=3$ ( $p$ and $u$ are measured in units of $\left.\Lambda, u=3 \pi^{2} 10^{-5}, r_{\mathrm{c}} \simeq 6.802854731032857, \alpha=2.25\right)$. (a) dimensionful function $Y(p, 0)+1=\Gamma^{(2)}(p, 0) / p^{2}$; (b) same data as (a). Top panel: $\Gamma^{(2)}(p, 0) / p^{2} \sim p^{-\eta}$ with $\eta \simeq 0.041$ for $p \rightarrow 0$ (red dashed line). Bottom panel: expected UV scaling for the self-energy; the dashed line shows the exact two-loop result for the slope.

tion of the quality of the present approximation. To this end, we use the family of cut-off functions $R_{k}\left(q^{2}\right)=$ $\alpha Z_{k} q^{2} /\left(\exp \left(q^{2} / k^{2}\right)-1\right)$ and vary systematically the parameters $\alpha, \tilde{p}_{0}$, and $\tilde{\rho}_{0}$. In all cases studied, we find the dependence on $\tilde{p}_{0}$ and $\tilde{\rho}_{0}$ to be much smaller than that on $\alpha$, so that only the latter needs to be considered. As a function of $\alpha$, physical quantities typically exhibit a single extremum $\alpha^{*}$, located near $\alpha=2$, which moreover, always points towards the best numerical estimates. Following the principle of minimal sensitivity (PMS) [12], we regard these extremum values, being locally independent of $\alpha$, as our best values.

We now turn to the discussion of results obtained, at criticality, first in dimension $d=3$ and for various values of $N$. The initial condition of the flow is taken to be $\Gamma_{\Lambda}^{(2)}(p, \rho)=p^{2}+r+u \rho$, where the bare coupling $u$ sets a scale, $[u]=[p]$, independent of the cut-off $\Lambda$. Keeping $u$ fixed, the critical value $r=r_{\mathrm{c}}$ is found by dichotomy. All expected features of $\Gamma_{k}^{(2)}(p)$ at criticality are observed, as illustrated in Fig. 1] Fig. 17 shows the typical shape of $Y(p, 0)$. In the infrared (IR) regime $k \ll p \ll u, \Gamma_{k}^{(2)}(p, 0)=p^{2}(Y(p, 0)+1) \sim p^{2-\eta}$, (Fig. 1 1 b, upper panel). This IR behavior of $\Gamma_{k}^{(2)}(p)$ can be used to extract the value of $\eta$; the value thus obtained is in excellent agreement with that deduced directly from the renormalization condition. The ultraviolet (UV) regime $k, u \ll p \ll \Lambda$ exists if $u$ is sufficiently small; this regime can be studied perturbatively and one finds that, in leading order, $p^{2} Y(p, 0) \sim u^{2} \log (p / u)$. The present approximation reproduces this logarithmic behaviour with, however, a prefactor $8 \%$ larger than the two-loop result (Fig. 1b, lower panel). Note that the complete two-loop behavior can be recovered by a simple improvement of the BMW scheme [10].

A quantity particularly sensitive to the UV-IR crossover region is the shift, due to interactions, of the critical temperature of the dilute Bose gas [13]. In the limit of small coupling, the shift is proportional to $u$. The proportionality coefficient is given (with the normalization used in Ref. [13]) by the non-universal quantity

$$
c=\left[-\frac{256}{u N} \zeta[3 / 2]^{-\frac{4}{3}} \int \mathrm{d}^{3} p\left(\frac{1}{\Gamma^{(2)}(p)}-\frac{1}{p^{2}}\right)\right],
$$

in the limit $u \rightarrow 0$. Note that the integrand is peaked at values of $p \sim u$, and is significant for momenta typically in the range $[N u / 100,10 N u]$. Initially introduced for $N=2$, corresponding to Bose-Einstein condensation, $c$ is often used as a sensitive benchmark of various approximations, as it tests the 2-point function over a wide range of momenta. It has been computed, for several values of $N$, on the lattice and with high-order (six-loop) perturbation theory (see Table I).

Table @ contains our results for $c$ and the critical exponents $\eta, \nu$ and $\omega$, together with some of the best estimates available in the literature. Our numbers are all given for the PMS values $\alpha^{*}$ of the cut-off parameter, and the digits quoted remain stable when $\alpha$ varies in the range $\left[\alpha^{*}-\frac{1}{2}, \alpha^{*}+\frac{1}{2}\right]$. The quality of these numbers is obvious: For all $N$ values where six-loop resummed calculations exist, our results for $c$ are within the error bars (and comparable to those obtained from an approximation specifically designed for this quantity [16]); the results for $\nu$ agree with previous estimates to within less than a percent, for all $N$; as for the values of $\eta$ and $\omega$, they are typically at the same distance from the Monte-Carlo and temperature series estimates as the results from resummed perturbative calculations. For $N=100$, we find $c=2.36, \eta=0.0023$, and $\nu=0.990$, which compare well to the exact large $N$ value $c \simeq 2.33$ [13] and to the values $\eta=0.0027$ and $\nu=0.989$ obtained in the $1 / N$ expansion [15]. Our numbers also compare favorably with those obtained at order $\partial^{2}$ in the DE scheme [8].

The two-dimensional case, for which exact results exist, provides an even more stringent test of the BMW scheme. We focus here on the Ising model $N=1$ which exhibits a standard critical behavior in $d=2$, and the corresponding critical exponents. (The coefficient $c$ is not defined in $d=2$.) The perturbative method that works well in $d=3$ fails here: for instance, the fixed-dimension expansion that provides the best results in $d=3$ yields, in $d=2$ and at five loops, $\eta=0.145(14)$ [27] in contradiction with the exact value $\eta=\frac{1}{4}$ [29]. We find instead $\eta=0.254, \nu=1.00$, and $\omega=1.28$ in excellent agreement with the exact values $\eta=\frac{1}{4}, \nu=1$ and the conjectured value $\omega=\frac{4}{3}$ [28].

To summarize, our results show that the single equation (5) (and its generalization to $O(N)$ models) is sufficient to obtain the momentum dependence of the two- 
TABLE I: Coefficient $c$ and critical exponents of the $O(N)$ models for $d=3$.

\begin{tabular}{|c|c|c|c|c|c|c|c|c|c|c|c|c|c|c|}
\hline \multirow[t]{2}{*}{$N$} & \multicolumn{4}{|c|}{ BMW } & \multicolumn{5}{|c|}{ Resummed perturbative expansions } & \multicolumn{5}{|c|}{ Monte-Carlo and high-temperature series } \\
\hline & $\eta$ & $\nu$ & $\omega$ & $c$ & $\eta$ & $\nu$ & $\omega$ & $c$ & Ref. $^{a}$ & $\eta$ & $\nu$ & $\omega$ & $c$ & Ref. $^{a}$ \\
\hline 0 & 0.034 & 0.589 & 0.83 & & $0284(25)$ & $0.5882(11)$ & $0.812(16)$ & & [17] & $0.030(3)$ & $0.5872(5)$ & 0.88 & & {$[18][19$} \\
\hline 1 & 0.039 & 0.632 & 0.78 & 1.15 & $0.0335(25)$ & $0.6304(13)$ & $0.799(11)$ & $1.07(10)$ & {$[17][14]$} & $0.0368(2)$ & $0.6302(1)$ & $0.821(5)$ & $1.09(9)$ & {$[20][21$} \\
\hline 2 & 0.041 & 0.674 & 0.75 & 1.37 & $0.0354(25)$ & $0.6703(15)$ & $0.789(11)$ & $1.27(10)$ & [17][14] & $0.0381(2)$ & $0.6717(1)$ & $0.785(20)$ & $1.32(2)$ & {$[22][23$} \\
\hline 3 & 0.040 & 0.715 & 0.73 & 1.50 & $0.0355(25)$ & $0.7073(35)$ & $0.782(13)$ & $1.43(11)$ & [17][14] & $0.0375(5)$ & $0.7112(5)$ & 0.773 & & 25 \\
\hline 4 & 0.038 & 0.754 & 0.72 & 1.63 & $0.035(4)$ & $0.741(6)$ & $0.774(20)$ & $1.54(11)$ & [17][14] & $0.0365(10)$ & $0.749(2)$ & 0.765 & $1.6(1)$ & {$[25][2$} \\
\hline 10 & 0.022 & 0.889 & 0.80 & & 0.024 & 0.859 & & & [26] & & & & & \\
\hline
\end{tabular}

${ }^{a}$ The first reference is for the critical exponents, the second for $c$.

point function with excellent accuracy, in all momentum regimes, for all $N$, and in any dimension. All this is obtained at a modest numerical cost using simple numerical techniques. The study presented here is only the leadingorder of a systematic approximation scheme. A study of the higher orders would be necessary in order to quantify the accuracy that has been reached. However, the robustness of our results can already be gauged from the weak residual dependence on the cut-off function.

We focused here on critical theories since numerous and accurate results exist for the critical regime, allowing for detailed and systematic checks, but it is clear that the method can be also used to deal with generically simpler situations. For instance, one could calculate the structure factor as a function of the momentum and the correlation length, which is of experimental interest. The effect of an external magnetic field could also be investigated by taking advantage of the built-in field dependence of $\Gamma_{k}^{(2)}$. A detailed investigation of the $d=2, N>1$ cases, is also at hand. Finally, this approach is not limited to $O(N)$ theories. It can also be applied to disordered, nonequilibrium, or quantum systems, expanding from existing studies within the DE scheme of, e.g. absorbing phase transitions [4, 7], or random-field models [30].

We acknowledge support from the ECOS project \#U05E01 and the PEDECIBA program (Uruguay). FB, RMG, and NW thank LPTMC (Paris) for hospitality.

[1] C. Wetterich, Phys. Lett. B 301, 90 (1993).

[2] J. Berges, N. Tetradis, and C. Wetterich, Phys. Rep. 363, 223 (2002).

[3] S. Seide and C. Wetterich, Nucl. Phys. B 562, 524 (1999).

[4] L. Canet, H. Chaté, and B. Delamotte, Phys. Rev. Lett. 92, 255703 (2004).

[5] J.-P. Blaizot, R. Méndez-Galain, and N. Wschebor, Phys. Lett. B 632, 571 (2006).

[6] B. Delamotte, D. Mouhanna, and M. Tissier, Phys. Rev. B 69, 134413 (2004).

[7] L. Canet, et al., Phys. Rev. Lett. 95, 100601 (2005).

[8] L. Canet, et al., Phys. Rev. D 67, 065004 (2003); Phys. Rev. B 68, 064421 (2003).
[9] For an $N$-component field $\vec{\phi}$, Eq. (3) involves a trace on the field index and there are two kinds of two-point functions, the transverse and the longitudinal ones [11].

[10] F. Benitez, et al., in preparation.

[11] J. P. Blaizot, R. Méndez-Galain, and N. Wschebor, Eur. Phys. J. B 58, 297 (2007); F. Benitez, R. Méndez-Galain, and N. Wschebor, Phys. Rev. B 77, 024431 (2008).

[12] P. Stevenson, Phys. Rev. D 23, 2916 (1981).

[13] G. Baym, et al., Phys. Rev. Lett. 83, 1703 (1999); G. Baym, J-P. Blaizot, and J. Zinn-Justin, Europhys. Lett. 49, 150 (2000).

[14] B. M. Kastening, Phys. Rev. A 69, 043613 (2004).

[15] M. Moshe and J. Zinn-Justin, Phys. Rep. 385, 69 (2003).

[16] J.-P. Blaizot, R. Méndez-Galain, and N. Wschebor, Phys. Rev. E 74, 051116 (2006); Ibid. 74, 051117 (2006).

[17] R. Guida and J. Zinn-Justin, J. Phys. A 31, 8103 (1998).

[18] P. Grassberger, P. Sutter, and L. Schäfer, J. Phys. A 30, 7039 (1997).

[19] A. Pelissetto and E. Vicari, Phys. Rep. 368, 549 (2002).

[20] Y. Deng and H. W. J. Blöte, Phys. Rev. E 68, 036125 (2003).

[21] X. Sun, Phys. Rev. E 67, 066702 (2003).

[22] M. Campostrini, et al., Phys. Rev. B 74, 144506 (2006).

[23] P. Arnold and G. Moore, Phys. Rev. Lett. 87, 120401 (2001); see also V.A. Kashurnikov, N. V. Prokof'ev, and B. V. Svistunov, Phys. Rev. Lett. 87, 160601 (2001), where the estimate of $c$ is consistent with the value quoted here.

[24] M. Campostrini, et al., Phys. Rev. B 65, 144520 (2002).

[25] M. Hasenbusch, J.Phys. A 34, 8221 (2001).

[26] S. A. Antonenko and A. I. Sokolov, Phys. Rev. E 51, 1894 (1995).

[27] A.A. Pogorelov and I.M. Suslov, JETP 105, 360 (2007).

[28] J. Zinn-Justin, Quantum Field Theory and Critical Phenomena, (Clarendon Press, Oxford, 2002), p. 702.

[29] It has been conjectured (see [19] and references therein), and this is confirmed by $1 / N$ calculations, that the presence of non-analytic terms in the flow of the $\phi^{4}$ coupling $u$ could be responsible for the discrepancy between exact and perturbative results in $d=2$. According to Sokal, no problem should arise when all couplings, including the irrelevant ones, are retained in the RG flow, as done here. This probably explains the quality of our results in $d=2$.

[30] G. Tarjus and M. Tissier, Phys. Rev. Lett. 93, 267008 (2004); Phys. Rev. B 78, 024203 (2008); M. Tissier and G. Tarjus, Phys. Rev. B 78, 024204 (2008). 